\title{
Saúde ocupacional e riscos psicossociais em trabalhadores da limpeza de instituição de ensino superior: Um estudo qualitativo em Brasília, DF.
}

\author{
Occupational health and psychosocial risks in cleaning \\ workers at a university: a qualitative study in Brasília, \\ DF.
}

\section{Salud ocupacional y riesgos psicosociales en trabajadores de una universidad: un estudio cualitativo en Brasília, DF.}

\author{
Matheus de Oliveira ANDRADE \\ Vinícius Santos da CUNHA ${ }^{2}$ \\ Wallisson de Medeiros Sales LINS ${ }^{3}$ \\ Felipe Rodrigues YUNG ${ }^{4}$ \\ José Alberto Souza ABDON ${ }^{5}$ \\ Elza Maria de SOUZA ${ }^{6}$
}

RESUMO: O objetivo deste estudo foi analisar a percepção dos funcionários da limpeza em uma instituição pública de ensino superior sobre condições de trabalho e saúde no meio universitário, comênfase para os aspectos físico-ambientais e psicossociais que determinam a saúde deste grupo no ambiente onde estão inseridos. Foi conduzida uma pesquisa qualitativa, utilizando-se a técnica de entrevista em profundidade junto a 16 funcionários da limpeza de ambos os sexos. Os trabalhadores têm a percepção de que não são devidamente valorizados. A maioria afirmou que o trabalho influencia negativamente a saúde, principalmente devido a dores osteomusculares, alergia aos produtos químicos de limpeza, exaustão e estresse pela sobrecarga de trabalho e falta de colaboração da comunidade universitária. Além disso, todos possuem percepção negativa da terceirização, em decorrência da alta rotatividade de empresas que inviabiliza o cumprimento de direitos trabalhistas, como férias regulares e décimo terceiro salário. Conclui-se que os trabalhadores

\footnotetext{
1 Faculdade de Medicina, Universidade de Brasília. E-mail: matheus101995@gmail.com

2 Faculdade de Medicina, Universidade de Brasília. E-mail: viniciusantoscunha@yahoo.com.br

3 Faculdade de Medicina, Universidade de Brasília. E-mail: wallissonlins2011@gmail.com

4Faculdade de Medicina, Universidade de Brasília. E-mail: felipeyung95@gmail.com

5 Faculdade de Medicina, Universidade de Brasília. E-mail: betoabdon@hotmail.com

6 Departamento de Saúde Coletiva, Faculdade de Ciências da Saúde, Universidade de Brasília. E-mail: elzacer-

rado@gmail.com
} 
da limpeza estão submetidos a condições desfavoráveis de trabalho, de modo que a consolidação de um ambiente universitário promotor de saúde para o trabalhador pressupõe uma construção conjunta que envolva o trabalhador, o âmbito comunitário e o administrativo. Faz-se necessário o desenvolvimento de modelos de promoção de saúde para os trabalhadores terceirizados no ambiente estudado.

Palavras-chave:Saúde ocupacional; Risco psicossocial; Universidade.

ABSTRACT: The aim of this study was to analyze the perception of cleaning workers at a public institution of higher education on working and health conditions at the university environment,with emphasis on the physical-environmental and psychosocial factors that determine the health of this group in the environment where they work.A qualitative study was conducted, using the technique of in-depth interview with 16 workers of both genders. The workers have the perception that they are not properly valued.Most respondents stated that their type of work negatively influences their health, regarding musculoskeletal pain, allergic reactions to the chemical products, tiredness and stress by work overload and by the lack of collaboration of the university community. In addition, all of them have a negative perception of outsourcing, due to high turnover of companies, which prevents fulfillment of labor rights, such as regular vacations and Christmas bonus salary. In conclusion, cleaning workers are subjected to unfavorable working conditions, and consolidation of a health promoting university environment for the workers assumes an interaction between the worker, the community and the administrative level. The development of a health promotion model is mandatory for outsourced workers in the studied environment.

Keywords: Occupational health;Psychosocial risk; University.

RESUMEN: El objetivo de este estudio fue analizar la percepción de los trabajadores de la limpieza en una institución pública de educación superior, acerca de sus condiciones de trabajo y salud en el ámbito universitario, con énfasis en los factores físico-ambientales y psicosociales que determinan la salud de este grupo en el entorno en que trabajan. Se realizó un estudio cualitativo, utilizando la técnica de entrevista en profundidad con 16 trabajadores de ambos sexos. Los trabajadores tienen la percepción de que no se valoran adecuadamente.La mayoría de los encuestados afirmaron que su tipo de trabajo influye negativamente en su salud, con respecto a dolores músculos esqueléticos, reacciones alérgicas a los productos químicos, cansancio y estrés, por la sobrecarga de trabajo y por la falta de valorización y colaboración de la comunidad universitaria. Además tenían una percepción negativa de la contratación externa, debido a la alta rotación de las empresas, lo que impide el cumplimiento de los derechos laborales, como las vacaciones regulares y el aguinaldo. Se concluye que la consolidación de un entorno universitario promotor de salud para los trabajadores presupone una interacción entre el trabajador, la comunidad y el nivel administrativo. Es necesario el desarrollo de modelos de promoción de la salud para los trabajadores subcontratados en el entorno estudiado.

Palabras clave: Salud Ocupacional; Riesgo psicosocial;Universidad. 


\section{INTRODUÇÃO}

Diversos grupos de trabalhadores estão expostos a condições de trabalho desfavoráveis, as quais podem se associar a riscos ocupacionais no âmbito físico e psicossocial ${ }^{1}$. Tal aspecto foi evidenciado em estudo qualitativo de Glina et al. $(2001)^{2}$, no qual as situações de trabalho foram analisadas considerando dados obtidos dos prontuários médicos de trabalhadores de diversos setores. Os casos estudados foram caracterizados por: condições de trabalho danosas, dificuldades referentes à organização do trabalho e gestão ineficiente de pessoal. Esses achados corroboram os resultados de um estudo de revisão bibliográfica em que Camelo \& Angerami (2008) ${ }^{3}$ identificaram diversas classes de riscos psicossociais relacionados ao trabalho, tais como suporte ineficiente para a resolução de problemas, ambiguidade e conflito de papéis, ausência de controle sobre o trabalho, relacionamento interpessoal escasso, monotonia das atividades e sobrecarga de trabalho.

Os riscos psicossociais no trabalho englobam diversas dimensões e podem ser caraterizados, de forma geral, como riscos para a saúde física e mental decorrentes das condições físicas, sociais e organizacionais de trabalho ${ }^{4}$. Neste sentido, evidencia-se a importância da participação das instituições na constituição de estratégias para identificar e controlar os riscos psicossociais presentes nas atividades laborais para prevenção dos agravos à saúde delas derivados ${ }^{3}$.

Diversas classes de trabalhadores têm sido alvo de estudos relacionando condições de trabalho com a saúde física e mental dos trabalhadores. Alguns autores têm se interessado particularmente em estudar as consequências do trabalho de limpeza sobre a saúde dos trabalhadores dessa área em diversos ambientes ${ }^{5-8}$. Há correlação da atividade de limpeza com aumento do risco de doenças respiratórias ${ }^{9}$ e dermatológicas ${ }^{10}$ pelas características do ambiente de trabalho e exposição aos produtos químicos de limpeza, desordens osteomusculares ${ }^{6,11}$, além do aumento do risco de prejuízo na saúde mental destes trabalhadores ${ }^{12,13}$.

Em estudo descritivo feito com profissionais da limpeza urbana, Barbosa et al. $(2010)^{14}$ delinearam o perfil de bem-estar psicológico de trabalhadores de limpeza urbana, demonstrando uma redução nítida do interesse, motivação e esforço do trabalhador em buscar oportunidades para conseguir realizar seus objetivos, sugerindo o desenvolvimento de um quadro de deterioração psicológica nos funcionários estudados.

Dantas \& Dantas (1998) ${ }^{15}$ realizaram uma pesquisa qualitativa que avaliou a percepção das condições de trabalho dos funcionários de limpeza em uma instituição de ensino superior do nordeste brasileiro, promovendo uma reflexão acerca da relação estabelecida entre os trabalhadores e o ambiente físico, institucional e social de uma universidade. Em termos gerais, essa pesquisa constatou a falta de utilização de equipamentos de proteção individual, a precariedade dos ambientes de trabalho, a baixa qualidade de vida dos trabalhadores da limpeza entrevistados e o fato de haver pouca integração entre a empresa empregadora e seus funcionários. Tais achados sugerem a interação de uma variedade de determinantes, tanto ambientais quanto psicossociais, causadores de 
dano à saúde do trabalhador da limpeza.

Uma revisão sistemática acerca dos fatores psicossociais de risco no trabalho evidenciou a necessidade de mais pesquisas qualitativas na área, no sentido de conferir maior profundidade aos dados obtidos e promover uma aproximação à realidade dos trabalhadores estudados ${ }^{4}$. Considerando este aspecto e a escassez de trabalhos evidenciando a percepção dos funcionários da limpeza inseridos no ambiente universitário, o objetivo do presente estudo foi analisar a percepção que os funcionários da limpeza têm em relação às suas condições de trabalho e saúde no ambiente de uma instituição pública de ensino superior. Dessa forma, buscou-se enfatizar os aspectos físicoambientais e psicossociais que determinam, sob a ótica do trabalhador, a saúde deste grupo no ambiente onde estão inseridos.

\section{MÉTODO}

Trata-se de uma pesquisa qualitativa, na qual foi utilizada a técnica de entrevista em profundidade junto aos trabalhadores da limpeza da Faculdade de Ciências da Saúde e de Medicina da Universidade de Brasília (UnB), vinculados a uma empresa terceirizada prestadora de serviços de limpeza à universidade.

Foram entrevistados 16 trabalhadores, durante o primeiro trimestre de 2013, dos quais 12 eram do gênero feminino e 4 do masculino, com idades variando entre 25 e 62 anos. Para a escolha dos entrevistados, recorreu-se a estratégia definida por Sarriá et al. (1999) ${ }^{16}$ como um processo de amostragem casual em que os entrevistados são selecionados de acordo com a acessibilidade e a disponibilidade em contribuir com a pesquisa. Como critério de inclusão, foram selecionados indivíduos com mais de 3 anos de trabalho na área da limpeza.

As entrevistas foram norteadas por um roteiroelaborado pelo grupoproponente dessa pesquisa (Quadro 1 do Anexo) no formato estruturado, isto é, contendo questionamentos gerais que poderiam ser aprofundados pelo próprio entrevistador no momento da entrevista ${ }^{17}$. As entrevistas foram gravadas com o consentimento dos entrevistados e transcritas verbatim para análise temática e de conteúdo.

Os dados foram analisados seguindo-se uma abordagem dos grandes temas pré-estabelecidos no roteiro de perguntas, os quais foram desdobrados em subtemas e derivações de acordo com as respostas dos entrevistados, conforme demonstrado no Quadro 2. 
Quadro 2: Sistematização dos grandes temas e principais aspectos abordados nas entrevistas

\begin{tabular}{|c|c|}
\hline Grandes temas & Subtemas e derivações \\
\hline Percepção do estado de saúde & $\begin{array}{l}\text { - Bom estado de saúde (14)* } \\
\text {-Ressalvas quanto a: } \\
\text { Dores osteomusculares (6) } \\
\text { Alergia aos produtos (2) } \\
\text { Hipertensão arterial (1) } \\
\text { - Péssimo estado de saúde (2) } \\
\text { - Alergia aos produtos (1) } \\
\text { - Dores osteomusculares (1) }\end{array}$ \\
\hline Relação entre o trabalho e saúde & $\begin{array}{l}\text { - Trabalho influencia saúde: (11) } \\
\text { - Dores osteomusculares (5) } \\
\text { - Alergia (3) } \\
\text { - Cansaço excessivo (2) } \\
\text { - Trabalho não influencia saúde (5) }\end{array}$ \\
\hline Relação entre o trabalho e estresse & $\begin{array}{l}\text { - Trabalho causa estresse (12) } \\
\text { - Falta de colaboração da comunidade universitária (7) } \\
\text { - Carga horária (2) } \\
\text { - Volume de trabalho (1) } \\
\text { - Trabalho não causa estresse (4) }\end{array}$ \\
\hline $\begin{array}{l}\text { Influência da estrutura física do } \\
\text { ambiente de trabalho }\end{array}$ & $\begin{array}{l}\text { - Estrutura influencia negativamente (11) } \\
\text { - Infiltrações e vazamentos (7) } \\
\text { - Sala de repouso inadequada (5) } \\
\text { - Descargas dos sanitários não funcionam (1) } \\
\text { - Estrutura não influencia (5) }\end{array}$ \\
\hline $\begin{array}{l}\text { Adoção de práticas saudáveis na } \\
\text { realização do trabalho }\end{array}$ & $\begin{array}{l}\text { - Adota (9) } \\
\text { - Não adota (7) } \\
\text { - Falta de equipamentos de proteção individual (5) } \\
\text { - Postura corporal incorreta (3) }\end{array}$ \\
\hline $\begin{array}{c}\text { Relação com a comunidade } \\
\text { universitária }\end{array}$ & $\begin{array}{l}\text { - Boa relação (15) } \\
\text { - Ressalvas quanto a: } \\
\text { Falta de colaboração da comunidade universitária (7) } \\
\text { - Não se relaciona com as pessoas (1) }\end{array}$ \\
\hline $\begin{array}{l}\text { Percepção da importância } \\
\text { da atividade de limpeza para a } \\
\text { universidade }\end{array}$ & $\begin{array}{l}\text { - Atividade é importante (16) } \\
\text { - Ressalvas quanto à falta de valorização por parte da comunidade universitária } \\
\text { (10) }\end{array}$ \\
\hline $\begin{array}{l}\text { Sugestões para facilitar a } \\
\text { execução das tarefas }\end{array}$ & $\begin{array}{l}\text { - Ações básicas de higiene (7) } \\
\text { - Não intervenção da comunidade universitária durante a realização das lavagens } \\
\text { (3) } \\
\text { - Respeito com os trabalhadores (3) } \\
\text { - Reivindicação política de melhores condições por parte dos estudantes (3) } \\
\text { - Melhor utilização do espaço físico dos centros acadêmicos (2) }\end{array}$ \\
\hline $\begin{array}{l}\text { Percepção dos funcionários sobre a } \\
\text { empresa empregadora }\end{array}$ & $\begin{array}{l}\text { - Ausência de treinamento de capacitação e educação continuada (16) } \\
\text { - Ausência de ações benéficas para a saúde (12) } \\
\text { - Fornecimento inadequado de equipamentos de proteção individual (12) } \\
\text { - Ausência de práticas de ginástica laboral (6) } \\
\text { - Ausência de plano de saúde (2) } \\
\text { - Orientação sobre segurança do trabalho (7) } \\
\text { - Adoção de medidas benéficas (4) } \\
\text { - Fornecimento adequado de equipamentos de proteção individual (4) }\end{array}$ \\
\hline $\begin{array}{c}\text { Terceirização do serviço de limpeza } \\
\text { na universidade }\end{array}$ & $\begin{array}{l}\text { - Avaliação negativa da terceirização (16) } \\
\text { - Rotatividade de empresas (16) } \\
\text { - Ausência de férias regulares (16) } \\
\text { - Instabilidade no emprego (3) } \\
\text { - Baixo salário (1) }\end{array}$ \\
\hline
\end{tabular}

*Os números entre parênteses mostram a quantidade de entrevistados, dentro do total de 16, que relataram o determinado 
tema durante a entrevista.

O presente estudo foi aprovado pelo Comitê de Ética em Pesquisa da FS-UnB.

\section{RESULTADOS}

\section{Relação entre trabalho, danos ocupacionais e saúde}

A maioria dos entrevistados relatou bom estado de saúde, embora quando questionados sobre a relação entre trabalho e saúde a maioria afirmou que o trabalho influencia negativamente a saúde, principalmente no que se refere a dores osteomusculares, alergia aos produtos químicos de limpeza e hipertensão arterial, como mostrado na seguinte passagem:

"É um trabalho que causa muito problema de coluna porque é um serviço que força muito. Passei a ter dores de coluna a partir do momento em que comecei a trabalhar como funcionário de limpeza." (Entrevista 4, Linhas 12-14)

Também foram relatados: exaustão pela sobrecarga de trabalho e pelo tempo de deslocamento casa-trabalho, além de alteração no horário de sono.

Deve-se destacar que muitos dos entrevistados que afirmaram possuir um bom estado de saúde não realizavam acompanhamento médico regular, como ilustrado pela citação:

"Não sinto nada, me sinto bem. Mas nunca fiz exame nenhum. Assim, preciso fazer, mas nunca faço porque no público é mais difícil e pra pagar é muito caro." (Entrevista 2, Linhas 9-11)

Os trabalhadores referiram também a relação entre trabalho e estresse, principalmente devido à falta de colaboração da comunidade universitária, havendo também referências quanto ao volume de trabalho e carga horária como fatores desencadeantes de estresse, como observado na seguinte citação:

"Acho que a carga de trabalho influencia a saúde devido ao horário que a gente levanta para chegar aqui. Eu levanto quatro horas da manhã [...]. Acaba sendo um horário muito corrido..." (Entrevista 4, Linhas 8-10)

Diversos trabalhadores afirmaram que o estresse no trabalho influenciava negativamente outras áreas de sua vida, como o ambiente familiar. Associado a esse aspecto, observou-se uma tendência de isolamento social como forma de minimizar as consequências de tal estresse, como ilustrado no comentário abaixo:

“Às vezes eu fico nervosa em casa, aí o meu menino fala: 'Ah, mãe, você traz seus problemas e vem descontar na gente'. Mas aí eu vou fazer minhas coisas lá em casa e esqueço logo. [...] Com os funcionários não tem problema não, porque aí, nesse caso, eu me isolo para não brigar com 
ninguém". (Entrevista 3, Linhas 39-44)

De acordo com os entrevistados, a estrutura física da universidade influencia de forma negativa seu trabalho e sua saúde, principalmente devido à presença de infiltrações e vazamentos, precariedade e mau uso das instalações sanitárias, bem como inadequação do tamanho da sala de repouso para o número de trabalhadores existentes. Isso pode ser percebido a seguir, em que, mais uma vez, foi notória a tendência de isolamento como escape da condição de desgaste psicológico:

"Eu acho que por isso aqui ser uma faculdade deveria ter mais coisas... Nós só temos essa salinha e uma hora de almoço. Uma hora de almoço pra 26 pessoas esquentarem sua marmita no micro-ondas é muito pouco. Quase não descansa... [...] Essa salinha aí está muito apertada para nós porque é muita gente. Por isso é que eu me isolo. Tem um quartinho no segundo andar entre um banheiro e outro, aí eu fico lá. Eu tenho só uma hora de almoço e lá eu coloco um papelão no chão e me deito lá. Lá é mais calmo, não tem estresse" (Entrevista 3, Linhas 58-61 e 69-71)

Quanto à realização de praticas saudáveis no trabalho, como o uso de equipamentos de proteção individual (EPIs), mais da metade dos trabalhadores afirmou adotar tais práticas. Aqueles que relataram não adotar práticas saudáveis justificaram a não adoção principalmente pelo fornecimento precário de EPIs.

\section{Relação com a comunidade universitária}

Todos os entrevistados afirmaram que a atividade de limpeza é importante para o pleno funcionamento do ambiente universitário. Entretanto, constatou-se que os trabalhadores têm a percepção de que a comunidade universitária não compartilha da visão da importância do trabalhador de limpeza, uma vez que não é observada uma devida valorização por parte de alunos e funcionários.

A maioria dos entrevistados afirmou que tem boa relação com os estudantes e funcionários em geral. Entretanto, muitos trabalhadores ressaltaram que não há uma colaboração dos estudantes para a realização da limpeza. Também foi levantada a questão da existência de episódios em que alguns funcionários da administração da universidade apresentaram uma postura arbitrária e desrespeitosa em relação aos trabalhadores.

Segundo a percepção dos entrevistados, a comunidade universitária pode contribuir de diversas maneiras para melhorar as condições de trabalho e saúde dos funcionários da limpeza, principalmente através de medidas simples como a deposição do lixo nas lixeiras, a execução da descarga após uso dos sanitários, a organização do ambiente físico dos centros acadêmicos e o respeito ao trabalhador, como sintetizado na observação seguinte:

"Eles podiam compreender mais a gente, entender mais a nossa parte. Porque a gente faz muito para ganhar pouco. Muitas vezes são os alunos e os outros funcionários mesmo que acabam 
estragando tudo que a gente faz, como, por exemplo, jogando lixo no chão. Se todos colocassem o lixo na lixeira, como eu acho que é o certo, sairia bem mais fácil pra gente, ajudaria muito mais a gente." (Entrevista 4, Linhas 65-69)

Alguns entrevistados também enfatizaram que uma ação política por parte dos estudantes no sentido de reivindicar melhores condições para os trabalhadores da limpeza seria potencialmente benéfica.

\section{Percepção dos funcionários sobre a empresa empregadora}

A maioria dos entrevistados afirmou que a empresa para a qual eles prestam serviço não adota medidas benéficas em relação à saúde dos trabalhadores. Os principais aspectos ressaltados pelos funcionários se relacionam ao fornecimento precário de equipamentos de proteção individual e treinamento apropriado, à ausência de práticas de ginástica laboral e à falta de plano de saúde. A minoria referiu o fornecimento de equipamentos de proteção de maneira adequada. Todos os entrevistados afirmaram que não há um treinamento para a realização do trabalho, nem educação permanente, como é demonstrado no comentário a seguir:

"Muita gente entra sem saber como é feito o trabalho. [...] Têm muitas coisas ali que a gente não pode colocar a mão. E isso não é passado para o funcionário, você tem que se virar." (Entrevista 8, Linhas 137-139)

Segundo informações coletadas, a ginástica laboral chegou a ser fornecida para os trabalhadores da limpeza, mas eles atualmente não possuem acesso a essa atividade. Muitos desses profissionais manifestaram-se favoráveis à implementação da ginástica laboral como uma medida profilática, o que está resumido na observação seguinte:

"Aqui era para ter uma ginástica para nós pelo menos uma horinha, né? Uma época teve e muita gente passou a diminuir a solicitação de atestado por conta de problema na coluna. Não sei por que pararam de fornecer..." (Entrevista 3, Linhas 86-88)

Quando questionados acerca da existência de orientação sobre segurança do trabalho, afirmaram que há um técnico em segurança do trabalho da empresa responsável por exercer essa função. Um dos entrevistados referiu o pagamento, por parte da empresa, de adicional de insalubridade que, em sua percepção, não é suficiente.

\section{Percepção sobre a terceirização de serviços}

Constatou-se que todos os funcionários estão insatisfeitos com a terceirização do serviço de limpeza por parte da universidade. Isso decorre principalmente da alta rotatividade de empresas prestadoras de serviço, o que traz como consequência a falta de férias regulares, sentimento de instabilidade no trabalho, não pagamento de direitos trabalhistas durante as transições de empresas 
e baixo salário. Esses aspectos são ilustrados pelos seguintes excertos das entrevistas:

"A gente está inseguro, né... Como não sei se ela (empresa) vai ficar ou se ela vai sair, fico um pouco preocupada, com medo. Porque quando têm essas coisas de ficar saindo empresa, manda muita gente embora. Eu não confio nisso aqui”. (Entrevista 2, Linhas 186-189)

"É uma situação difícil. De uns tempos para cá tem mudado de empresa constantemente. A gente fica seis meses, um ano, muito tempo sem tirar férias, porque quando a gente vai tirar férias sai uma empresa e entra outra." (Entrevista 9, Linhas 103-105)

\section{DISCUSSÃO}

O estudo em foco apresenta limitações relativas à seleção casual da amostra, restrita a um ambiente universitário específico. Embora a pesquisa qualitativa não requeira aleatorização, esse processo, quando possível, minimiza possíveis vieses de seleção. No entanto, mais importante que a randomização é a escolha apropriada dos sujeitos para o aprofundamento do tema investigado ${ }^{17}$.

Os resultados obtidos sugerem que todos os entrevistados acreditam na importância do seu trabalho para a sociedade e para o ambiente em que estão inseridos. No entanto, as condições precárias para o desempenho das funções, associadas à falta de consciência da comunidade universitária, a terceirização e suas consequências são fatores geradores de desgaste físico e psicológico. Os achados também sugerem que isto leva à deterioração da saúde, interfere na relação familiar e favorece o isolamento social, resultando em um círculo vicioso, no qual um fator influencia negativamente o outro.

Esses aspectos estão em consonância com os resultados obtidos por Camelo \& Angerami $(2008)^{3}$ no que se refere ao relacionamento interpessoal escasso, à dúvida na carreira e à sobrecarga de trabalho. Em relação à deterioração psicológica, os resultados obtidos no presente estudo corroboram os resultados encontrados por Barbosa et al. $(2010)^{14}$, bem como os obtidos por Glina et al. $(2001)^{2}$ relativos à influência do trabalho nas condições de saúde física e mental. Além das condições de trabalho precárias proporcionadas pelo modelo contratual estabelecido, a própria atividade do funcionário de limpeza não fornece perspectivas de ascensão profissional, sendo este possivelmente um fator desestimulante que contribui para o quadro de deterioração psicológica.

Os achados também corroboram os obtidos por Dantas \& Dantas $(1998)^{15}$, no que se refere à precariedade dos ambientes de trabalho e da qualidade de vida dos trabalhadores em ambientes universitários, além da baixa integração entre a empresa empregadora e seus funcionários, sendo que o presente estudo evidenciou ainda uma possível influência da atitude da comunidade universitária e do modelo de terceirização na geração de estresse psicológico. É importante destacar que ambos os estudos foram realizados no meio universitário e, apesar de envolverem perspectivas diferentes, demonstraram que os trabalhadores da limpeza nesse ambiente são sujeitos a condições precárias 
de trabalho e saúde.

Monteiro et al. (2004) $)^{7}$ destacaram a importância da educação continuada para minimizar os riscos ocupacionais em um grupo de trabalhadores da limpeza terceirizados em um hospital universitário. Dessa forma, a ausência de treinamento adequado e educação continuada relatada pelos entrevistados é um fator de risco ocupacional adicional para os trabalhadores, evidenciando a necessidade de maior compromisso por parte da empresa em relação às condições de trabalho dos funcionários da limpeza para a proteção da saúde desses trabalhadores.

Um estudo realizado por Abbas et al. (2013) $)^{12}$ avaliou a saúde mental de trabalhadores da limpeza em quatro hospitais públicos no Egito, comparando trabalhadores temporários em relação aos efetivos com características sócio-demográficas semelhantes. Evidenciou-se maior prevalência de desordens mentais e abuso de drogas em funcionários temporários em comparação com os trabalhadores do quadro efetivo de funcionários da limpeza. Estes resultados podem ser correlacionados com os obtidos no presente estudo, considerando que a alta rotatividade de empresas terceirizadas reportada pelos trabalhadores avaliados promove um status de insegurança no trabalho equivalente aos trabalhadores informais temporários do estudo de Abbas et al. ${ }^{12}$, enfatizando o risco psicossocial do grupo em questão.

O modelo de terceirização do serviço de limpeza por parte da universidade deve ser revisto, considerando que a alta rotatividade de empresas causa um sentimento de insegurança no trabalhador, além de impedir que direitos trabalhistas mínimos, como férias regulares e décimo terceiro salário, sejam cumpridos adequadamente. A terceirização é descrita por Repullo (1997) ${ }^{18}$ como uma “tática de redução de custos pela exploração de precárias relações de trabalho"e há evidências crescentes de que este modelo afeta negativamente a segurança e saúde ocupacional ${ }^{19,20}$. Apesar da terceirização ser globalmente difundida nas sociedades industrializadas, ainda não há modelos consolidados de promoção de saúde voltados para este grupo de trabalhadores considerando suas peculiaridades ocupacionais.

Considerando os modelos teóricos a respeitodos fatores psicossociais de risco no trabalho, o modelo de desequilíbrio esforço-recompensa se aplica aos presentes resultados acerca da relação dos trabalhadores com a comunidade universitária. Tal modelo consisteno desbalanço entre esforço no trabalho e ganho, não somente no sentido de remuneração, mas também englobando o status de valorização e reconhecimento do trabalho realizado ${ }^{21}$. Sendo assim, o fato de que os trabalhadores têm a percepção de que não são devidamente valorizados por parte da comunidade universitária, a despeito do esforço para realização do trabalho, é um aspecto gerador de desgaste psicológico. Este achado é compatível com os resultados de Gonzales \& Carvalho (2003) ${ }^{22}$, que delinearam o perfil de saúde mental de trabalhadores do serviço da limpeza de um hospital universitário e determinaram que este grupo apresentava a percepção de desvalorização de seu trabalho e sentimento de invisibilidade social. 
Segundo Mello et al. $(2010)^{23}$, universidades constituem espaços sociais estratégicos para a promoção de saúde, pois podem contribuir para a saúde de grupos específicos a elas relacionados, com consequente impacto sobre a população geral. Contrariamente ao pressuposto de que em uma instituição de ensino superior de saúde os profissionais de limpeza teriam uma atenção especial por parte da comunidade acadêmica, verificou-se que esta contribui pouco ou quase nada para minimizar os malefícios laborais à saúde dessa categoria profissional. Dessa forma, os resultados demonstrados contradizem o conceito de ambiente universitário promotor de saúde.

Estudos realizados na área de educação médica demonstram que a relação entre o trabalho e o processo saúde-doença não tem sido contempladas de forma adequada na formação dos estudantes $^{24}$. Este cenário não é exclusivo do Brasil ${ }^{25}$ e cerca de um terço das escolas médicas dos Estados Unidos não desenvolvem o tema de saúde ocupacional no programa dos cursos de graduação ${ }^{26}$. Considerando tal precariedade da formação médica e de outros profissionais de saúde no âmbito da saúde ocupacional, a promoção de saúde do trabalhador no ambiente universitário deve ser incorporada nas faculdades de saúde para integrar os estudantes no cenário de prática desde o início dos cursos.

Levando em conta que a falta de colaboração e valorização por parte da comunidade universitária é um aspecto que prejudica as condições de trabalho e saúde dos funcionários, são necessárias medidas de conscientização da comunidade universitária, visando ao incentivo de práticas básicas de higiene, organização e respeito ao trabalhador da limpeza.

\section{CONSIDERAÇÕES FINAIS}

Embora com limitações, o presente estudo evidenciou que a realidade dos trabalhadores de limpeza merece uma atenção especial, assim como a integração entre contratante, contratada e comunidade universitária para a preservação e o aprimoramento da saúde dos referidos trabalhadores. Considerando os riscos físicos e psicossociais a que estão expostos, faz-se necessária a criação de ferramentas para a melhoria das condições de trabalho e desenvolvimento de modelos para fomentar a saúde dos trabalhadores terceirizados. Sendo assim,a promoção de saúde no ambiente universitário deve ser incorporada rotineiramente, principalmente nas faculdades de saúde, para dar coerência aos objetivos da educação profissional em saúde. Deve-se ressaltar a necessidade de estudos adicionais, envolvendo a categoria estudada e outras categorias profissionais para melhor entendimento da influência do trabalho na condição de saúde dos trabalhadores dentro das universidades.

\section{REFERÊNCIAS BIBLIOGRÁFICAS}

1. Carayon P, Smith MJ, Haims MC. Work organization, job stress, and work-related musculoskeletal disorders. Hum Factors. 1999; 41(4):644-63.

2. Glina DMR, Rocha LE, Batista ML, Mendonça MGV. Saúde mental e trabalho: uma 
reflexão sobre o nexo com o trabalho e o diagnóstico, com base na prática. Cad Saude Pública. 2001; 17(3): 607-616.

3. Camelo SHH, Angerami ELS. Riscos psicossociais no trabalho que podem levar ao estresse: uma análise da literatura. Cienc Cuid e Saúde. 2008; 7(2): 232-240.

4. Costa LS, Santos M. Fatores psicossociais de risco no trabalho: lições aprendidas e novos caminhos. Int J Working Conditions. 2013; 5: 39-58.

5. Alamgir H, Yu S. Epidemiology of occupational injury among cleaners in the healthcare sector. Occup Med (Lond). 2008; 58(6): 393-399.

6. Martarello, NA, Benatti MCC. Qualidade de vida e sintomas osteomusculares em trabalhadores de higiene e limpeza hospitalar. Rev Esc Enferm USP. 2009, 43(2): 422-428.

7. Monteiro MI, Chillida MSP, Bargas EB. Educação continuada em um serviço terceirizado de limpeza de um hospital universitário. Rev Lat Am Enfermagem. 2004; 12(3): 541-548.

8. Chillida MSP, Cocco, MIM. Saúde do trabalhador \& terceirização: perfil de trabalhadores de serviço de limpeza hospitalar. Rev Lat Am Enfermagem. 2004; 12(2): 271-276.

9. Vizcaya D, Mirabelli MC, Orriols R, Antó JM, Barreiro E, Burgos F, Arjona L, Gomez F, Zock JP. Functional and biological characteristics of asthma in cleaning workers. Respir Med. 2013; 107(5): 673-83.

10. 10. Bauer A. Contact dermatitis in the cleaning industry Curr Opin Allergy Clin Immunol. 2013; 13(5): 521-4.

11. Chang JH, Wu JD, Liu CY, Hsu DJ. Prevalence of musculoskeletal disorders and ergonomic assessments of cleaners. Am J Ind Med. 2012; 55(7): 593-604.

12. Abbas RA, Hammam RA, El-Gohary SS, Sabik LM, Hunter MS. Screening for common mental disorders and substance abuse among temporary hired cleaners in Egyptian Governmental Hospitals, Zagazig City, Sharqia Governorate. Int J Occup Environ Med. 2013; 4(1): 13-26.

13. Charles LE, Loomis D, Demissie Z. Occupational hazards experienced by cleaning workers and janitors: A review of the epidemiologic literature. Work. 2009; 34(1) 105-16.

14. Barbosa SC, Melo RLP, Medeiros MUF, Vasconcelos TM. Perfil de Bem-Estar Psicológico em Profissionais de Limpeza Urbana. Rev Psi: Org e Trab. 2010; 10(2): 54-66.

15. Dantas JAB, Dantas TMTA. Percepção das condições de trabalho dos funcionários responsáveis pela higiene e limpeza das instalações sanitárias: estudo de caso de uma instituição 
de ensino superior do nordeste - IES/NE, período março-abril /1997. Sitientibus. 1998; 18: 99116.

16. Sarriá A, Guardiã J, Freixa M. Introducción a la estadística en Psicología. $1^{\text {a }}$ Edição. Barcelona: Ediciones de la Universitat de Barcelona; 1999.

17. Minayo MCS. O desafio do conhecimento: pesquisa qualitativa em saúde. $8^{a}$ Edição. São Paulo, Rio de Janeiro: Hucitec, Abrasco; 2004.

18. Repullo Jr, R. Os sindicatos, a terceirização e a saúde dos trabalhadores. Rev Bras Saúde Ocup. 1997; 23(85/86): 79-82.

19. Mayhew C, Quinlan M, Ferris R. The effects of subcontracting/outsourcing on occupational health and safety: survey evidence from four Australian industries. Safety Science. 1997; 1(3): 163-78.

20. Mayhew C, Quinlan M. The effects of outsourcing on occupational health and safety: a comparative study of factory-based workers and outworkers in the Australian clothing industry. Int J Health Serv. 1999; 29(1) 83-107.

21. Reis ALPP, Fernandes SRP, Gomes AF. Estresse e fatores psicossociais. Psicol Cien Prof. 2010; 30(4): 712-725.

22. Gonzales BBA, Carvalho MDB. Saúde mental de trabalhadores do serviço de limpeza de um hospital universitário. Acta Scientiarum. 2003; 25(1): 55-62.

23. Mello ALSF, Moysés ST, Moysés SJ. A universidade promotora de saúde e as mudanças na formação profissional. Interface (Botucatu). 2010; 14(34): 683-92.

24. Dias EC, Silveira AM, Chiavegatto CV, Resende NP. O ensino das relações trabalhosaúde-doença na escola médica: percepçãoo dos alunos e proposta de aperfeiçoamento na UFMG. Rev Bras Educação Médica. 2006; 30(1): 20-26.

25. Goldman RH, Rosenwasser S, Armstrong E. Incorporating environmental/occupational medicine theme into the medical school curriculum. J Occup Med. 1999; 41(1): 47-52.

26. Levy B. The teaching of occupational health in American schools: five-year follow-up of an initial survey. Am J Public Health. 1985; 75(1): 79-80.

Artigo apresentado em 30-08-15

Artigo aprovado em 10-01-16 Artigo publicado no sistema em 30-03-16 


\section{ANEXO}

\section{Quadro 1: Roteiro para entrevista}

1. Como o(a) $\operatorname{Sr}($ a) considera o seu estado de saúde?

2. Qual é a sua opinião sobre a relação entre o seu trabalho e sua saúde?

3. Em sua opinião, existe relação entre o seu trabalho e estresse mental?

4. $\mathrm{O}(\mathrm{a}) \mathrm{Sr}(\mathrm{a})$ adota praticas saudáveis durante a realização do seu trabalho?

5. Fale-nos se o ambiente da universidade influencia a realização do seu trabalho.

6. Como é a sua relação com os estudantes da FS/FM-UnB?

7. Como é a sua relação com os funcionários da FS/FM-UnB?

8. Como você avalia a importância da sua atividade para a FS/FM-UnB?

9. $\mathrm{O}$ (a) $\mathrm{Sr}(\mathrm{a})$ acha que a comunidade universitária da FS/FM-UnB poderia contribuir de alguma forma para melhorar as suas condições de trabalho?

10. A empresa para a qual o(a) $\operatorname{Sr}($ a) presta serviços adota medidas para beneficiar a saúde dos trabalhadores?

11. Como é feito o treinamento de capacitação para a realização do seu trabalho?

12. O que o(a) $\operatorname{Sr}(a)$ pensa a respeito da terceirização do serviço de limpeza na UnB?

13. $\mathrm{O}(\mathrm{a}) \mathrm{Sr}(\mathrm{a})$ gostaria de fazer algum comentário a mais? 